\title{
Muonium diffusion dynamics in mercury oxide
}

\author{
J. Piroto Duarte ${ }^{\text {a,* }}$, J.M. Gil ${ }^{\text {a }}$, H.V. Alberto ${ }^{\text {a }}$, R.C. Vilão ${ }^{\text {a }}$, A. Weidinger ${ }^{\text {a }}$, \\ N. Ayres de Campos ${ }^{\mathrm{a}}$, S.F.J. Cox ${ }^{\text {b,c }}$, J.S. Lord ${ }^{\mathrm{c}}$, S.P. Cottrell ${ }^{\mathrm{c}}$, E.A. Davis ${ }^{\mathrm{d}}$ \\ ${ }^{a}$ Physics Department, University of Coimbra, P-3004-516 Coimbra, Portugal \\ ${ }^{\mathrm{b}}$ Physics Department, University College London, WC1E 6BT, UK \\ ${ }^{\mathrm{C}}$ ISIS Facility, Rutherford Appleton Laboratory, Chilton, Didcot, Oxon OX11 OQX, UK \\ ${ }^{\mathrm{d}}$ Department of Materials Science and Metallurgy, University of Cambridge, CB2 3QZ, UK
}

\begin{abstract}
The diffusion dynamics of the neutral muonium state found in $\mathrm{HgO}$ is addressed in this work. We propose a hopping model for the diffusion, and use it to analyse time-domain $\mu \mathrm{SR}$ data. It is found that the diffusion is an incoherent quantum process, with an activation energy of 5.2(2) meV. The analysis also points to the anti-bonding site as the best-suited candidate for the muon's localisation.

(C) 2005 Elsevier B.V. All rights reserved.
\end{abstract}

Keywords: II-VI Semiconductors; Mercury oxide; Muonium diffusion

\section{Introduction}

The understanding of the role of hydrogen in semiconductors has developed considerably in recent years since it was discovered that interstitial hydrogen could act as a shallow-donor impurity in compound semiconductors [1-4]. The $\mu$ SR technique played a crucial role in this discovery; indeed, the very first experimental evidence was obtained with $\mu$ SR experiments on cadmium sulphide and zinc oxide, where an unusually low hyperfine interaction associated with the muonium $(\mathrm{Mu})$ state was identified $[1,2]$. Since then, systematic surveys of other compound semiconductors, mostly oxides, have enlarged the list of host systems in which the shallow-level donor muonium state is formed $[5,6]$.

Within the context of a deep-level/shallow-level dual picture for hydrogen impurity states in semiconductors, the neutral muonium state found below $150 \mathrm{~K}$ in the wide-gap semiconductor mercury oxide appears somewhat as a further novelty $[7,8]$. Its hyperfine coupling parameters $A_{\text {iso }}=14.93 \mathrm{MHz}$ and $D=5.2 \mathrm{MHz}$ place it mid-way between the bond-centre muonium deep states known for long in the elemental and III-V compound semiconductors

\footnotetext{
*Corresponding author. Tel.: + 351239410685 ; fax: + 351239829158 .

E-mail address: piroto@ci.uc.pt (J. Piroto Duarte).
}

( $A_{\text {iso }}$ and $D \sim$ several tens of $\mathrm{MHz}$ ), and the anion antibonding shallow states recently discovered ( $A_{\text {iso }}$ and $D$ $\sim$ hundreds of $\mathrm{kHz}$ ). The uniqueness of this state arises from the unusual structure of $\mathrm{HgO}$, shaped by broken chains running parallel to the $a$-axis that coordinate the $\mathrm{Hg}$ and $\mathrm{O}$ atoms (see Fig. 1), contrasting with the much more regular tetrahedral coordination of most semiconductor systems. This makes controversial whether the neutral muonium state formed in $\mathrm{HgO}$ is located at the bondcentre or at the oxygen's anti-bonding site, and no experimental evidence clearing this matter has been put forward so far.

This neutral muonium state in $\mathrm{HgO}$ has also been found to undergo diffusion through crystallographically equivalent positions below ionisation. The existence of diffusion dynamics has been inferred from data taken with powder samples which imply averaging of the state's anisotropy [7]. In high-field TF geometry measurements, increasing temperature produces a gradual narrowing of the frequency powder-pattern lineshape characteristic of axially symmetric muonium; in LF repolarisation measurements, the $\Delta M=1$ level-crossing resonance dip narrows from an asymmetric powder-shape at low temperatures to a more symmetrical resonance at higher ones.

This work reports a quantitative $\mu \mathrm{SR}$ study performed on the diffusion dynamics of muonium in $\mathrm{HgO}$. Existing 

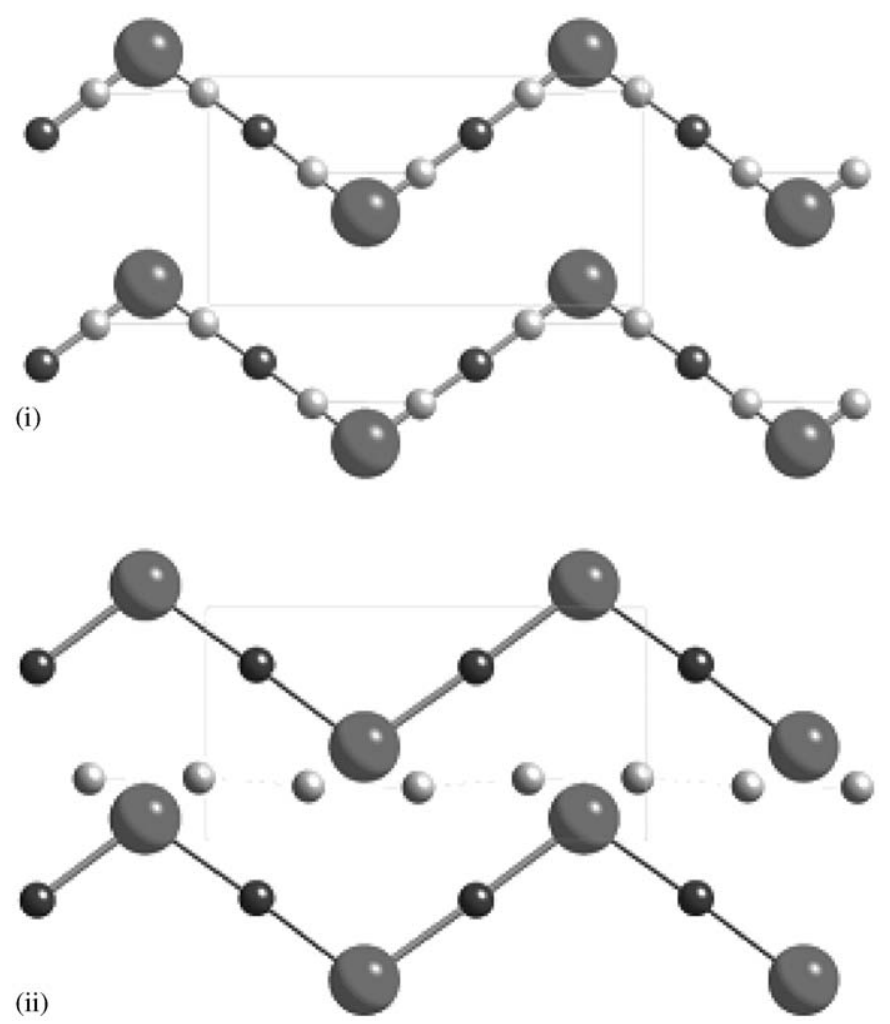

Fig. 1. The (i) bond-centre and (ii) anti-bonding diffusion paths considered in our model for muonium diffusion in $\mathrm{HgO}$. $\mathrm{Hg}$ atoms are represented by small, darker spheres, $\mathrm{O}$ atoms by large, brighter spheres and $\mathrm{Mu}$ by small white spheres.

and new experimental data taken on powder samples with the GPS (TF geometry) and EMU (LF geometry) spectrometers at PSI and ISIS respectively are analysed with a simplified hopping model, and its results are discussed.

\section{Diffusion model}

Presuming the observed muonium state has the same symmetry axis as the $\mathrm{Hg}-\mathrm{O}$ bond, hopping paths through bond-centre or anti-bonding sites in adjacent chains provide the anisotropy averaging observed in Ref. [7] (Fig. 1). Careful examination shows that in each path jumps take place between positions either with the same symmetry axis, or with symmetry axis concurrent at $107.3^{\circ}$, the $\mathrm{Hg}-\mathrm{O}-\mathrm{Hg}$ bond angle. For the sake of simplicity, one may start by assuming that all polarisations of the muonium system (muon and electron) do not suffer any change when it jumps between sites having the same symmetry axis. This reduces the hopping problem to the reversible transition between two muonium states with the same hyperfine parameters, but different symmetry axis, at a hopping rate $\Lambda$. We note that these considerations apply to both the bond-centre and the oxygen anti-bonding paths, and that therefore the model makes intrinsically no distinction between the two possibilities. We also make clear that the hopping rate $\Lambda$ refers to jumps between positions with different symmetry axis, i.e., every second position in the paths; the real hop rate will be the double of $\Lambda$.

The time evolution of the muon's polarisation in LF and TF geometries was computed using the equations of motion for all polarisations of two muonium systems with different hyperfine interaction tensors undergoing a reversible transition at a rate $\Lambda$ [9]. The procedure followed is a generalisation of the Wangsness-Bloch equations for a single muonium system to the two system case, adopting the remarks described in Ref. [10]. The two hyperfine tensors were constrained to have the same hyperfine parameters $A_{\text {iso }}=14.93 \mathrm{MHz}$ and $D=5.2 \mathrm{MHz}$, and symmetry axis concurrent at $107.3^{\circ}$. Powder averaging was performed numerically with the (also numerical) solutions of the two-muonium system equations. A phenomenological electronic depolarisation rate $v$ was included as well, in order to model the nuclear hyperfine interaction with the surrounding spin-carrying $\mathrm{Hg}$ nuclei $\left(17 \% S=\frac{1}{2}, 13 \% S=\frac{3}{2}\right)$.

Simulations performed with this model were found to reproduce the main features observed in the diffusion data above $10 \mathrm{~K}$, namely the expected onset of the powderdistribution narrowing in high TF and of the narrowing of the LF resonance dip to a symmetric shape at a jump rate near the value of the anisotropy parameter. This study also showed that the high-TF and LF repolarisation data should exhibit a very low sensitivity to the hop rate $\Lambda$ outside that region. It was found as well that the highest sensitivity to $\Lambda$ is achieved with data collected in the socalled $\mathrm{Mu}^{*} \mathrm{LF}$ magic field $[9,10]$, at which the amount of information regarding $\Lambda$ in the time spectra is maximal thanks to the non-existence of damping of the $f_{12}$ precession by the powder distribution.

\section{Results and discussion}

Most of the quantitative analysis with the diffusion model was performed on magic field time-spectrum data collected at the EMU spectrometer. Fig. 2 shows an Arrhenius plot of the temperature dependence extracted in that way for the Mu hop rate (double of $\Lambda$, see Section 2). Between 6 and $30 \mathrm{~K}$, it is in good agreement with the simple small-polaron model for incoherent quantum diffusion of light interstitials [11,12], bearing an activation energy of $E_{\mathrm{a}}=5.2(2) \mathrm{meV}$ and transition matrix element $J=0.065(3) \mathrm{meV}$. Such small activation energy confirms the quantum nature of the diffusion process, discarding classical "over-the-barrier" hopping. The transition matrix element $J$ is also within the expected range of values for insulators and semiconductors at low temperatures [12]. At $30 \mathrm{~K}$, the hop rate peaks; muonium is highly mobile at that temperature, tunneling about 800 times during its $2.2 \mu \mathrm{s}$ lifetime. Above $30 \mathrm{~K}$, the hop rate decreases, in what appears to be a change of diffusion regime. Trapping at a defect is not the mechanism responsible for this decrease, since the average length traveled by muonium at $30 \mathrm{~K}$ considering a one-dimensional random walk is only $60 \AA$, a 


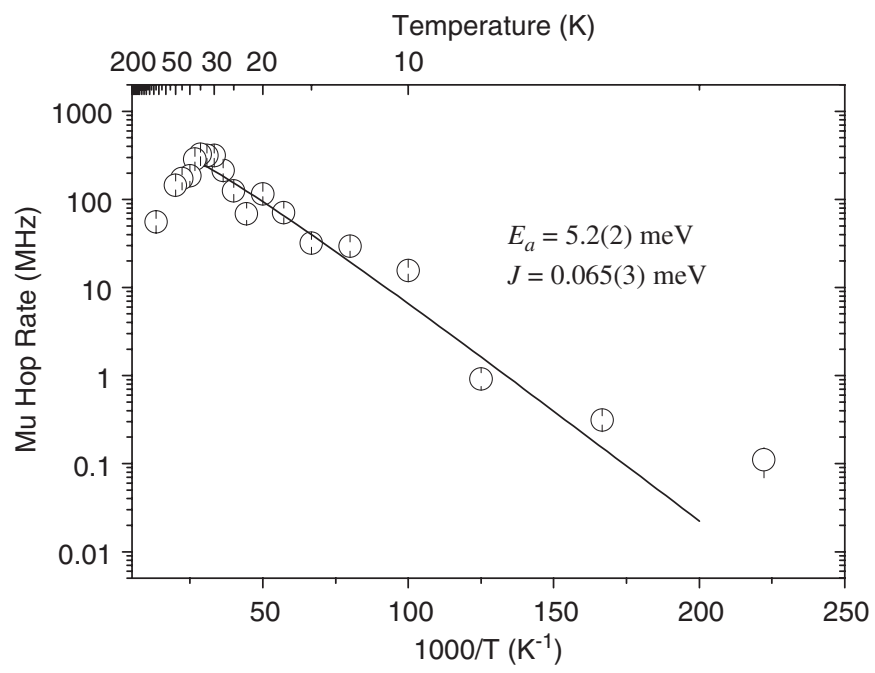

Fig. 2. Arrhenius plot of the Mu hop rate for the neutral muonium state in $\mathrm{HgO}$ up to $75 \mathrm{~K}$ (beyond this temperature, the effects of diffusion become masked due to ionisation of the muonium state). Bellow $30 \mathrm{~K}$, an incoherent quantum diffusion process is followed.

distance too small to allow muonium finding any impurity if one accounts for the high purity of the samples. Trapping at a grain boundary is ruled out in a similar way.

Insight about the muon site may also be derived from the magic-field analysis with the diffusion model. The electronic depolarisation rate $v$ was seen to increase with temperature from zero to $0.25(10) \mathrm{MHz}$ at $10 \mathrm{~K}$, decreasing thereof again to zero. Since $v$ is expected to model the nuclear hyperfine interaction with the $\mathrm{Hg}$ nuclei, its peak value may be taken as a guess for that interaction's coupling. A rough estimate of the spin-carrying $\mathrm{Hg}$ nuclei to muon distance assuming a $1 \mathrm{~s}$ type electronic wave function for the muonium computed with that coupling amounts to about $6 \AA$, a value far too large for the bond- centre site hypothesis, even taking into account that only one third of the $\mathrm{Hg}$ nuclei have spin. This is in agreement with recent theoretical results which find electrostatic stable positions for the muon at sites anti-bonding to the oxygen atom [13].

\section{Acknowledgements}

The assistance of all LMU staff at PSI and $\mu$ SR instrument scientists at ISIS is gratefully acknowledged. This work was performed at the Swiss Muon Source, Paul Scherrer Institute, Villigen, Switzerland, and partially supported by the European Commission under the Sixth Framework Programme through the Key Action: Strengthening the European Research Area, Research Infrastructures, contract no: RII3-CT-2004-505925, and the European Union Framework V access to Research Infrastructures Programme. Funding has also been provided by the EPSRC grants GMM0041 and GR/R25361 (UK) and the Portuguese Foundation for Science and Technology (FCT) through grant POCTI/35334/FIS/2000.

\section{References}

[1] J.M. Gil, et al., Phys. Rev. Lett. 83 (1999) 5294.

[2] S.F.J. Cox, et al., Phys. Rev. Lett. 86 (2001) 2601.

[3] M.H. Detlev, et al., Phys. Rev. Lett. 88 (2002) 045504.

[4] C.G. Van de Walle, Phys. Rev. Lett. 85 (2000) 1012.

[5] J.M. Gil, et al., Phys. Rev. B 64 (2001) 075205.

[6] S.F.J. Cox, et al., Physica B, this conference.

[7] J.M. Gil, et al., J. Phys.: Condens. Matter 13 (2001) L613.

[8] S.F.J. Cox, et al., J. Phys.: Condens. Matter 13 (2001) 9001.

[9] J. Piroto Duarte, M.Sc. Thesis, 2003.

[10] B.D. Patterson, Rev. Mod. Phys. 60 (1988) 69.

[11] H. Grabert, H.R. Schober, Hydrogen in metals III, in: H. Wipf (Ed.), Topics in Applied Physics, vol. 73, Springer, Berlin, 1997.

[12] V.G. Storchak, N.V. Prokof'ev, Rev. Mod. Phys. 70 (1998) 929.

[13] C.H. Park, private communication. 